Pacific

Journal of

Mathematics

\title{
A NOTE ON MURASUGI SUMS
}

ABIGAIL A. THOMPSON

Volume $163 \quad$ No. 2

April 1994 


\title{
A NOTE ON MURASUGI SUMS
}

\author{
Abigail Thompson
}

\begin{abstract}
We give two examples to show that the genus of knots is neither sub- nor super-additive under the Murasugi sum operation.
\end{abstract}

A number of "addition" operations can be defined on pairs of knots in $S^{3}$; the connected sum is the most obvious of these, but there are several other more complicated possibilities. A general question one can ask is: which properties of knots behave "nicely" under these operations? It has long been known that the genus of a knot is additive under connect sum. Schubert [Sc] showed that bridge number is additive minus one under connect sum.

Outstanding questions are how crossing number, unknotting number and tunnel number behave under connect sum. Only the most obvious inequalities are currently available, and they are quite weakfor example, the crossing number is obviously sub-additive, as is the unknotting number, and it is easy to show that the tunnel number of the connect sum of $K_{1}$ and $K_{2}$ is less than or equal to the sum of their tunnel numbers plus one.

A more complicated operation on pairs of knots is the band-connect sum. This operation is not well defined, since it depends on how the band is chosen. Gabai and Scharlemann simultaneously established the superadditivity of genus under band-connect sum [G1], [S].

Yet another operation combining knots is the Murasugi sum of two knots (see [G2] for a definition); this depends on a choice of Seifert surfaces for the knots as well as a choice of disks along which to do the sum. Gabai [G2] nevertheless has shown that under reasonable conditions many geometric properties of the Seifert surfaces are retained under the Murasugi sum. In particular, he has shown that the Murasugi sum of $K_{1}$ and $K_{2}$ along minimal genus Seifert surfaces $R_{1}$ and $R_{2}$ yields a minimal genus Seifert surface $R$ for the resulting knot $K$, so genus is additive under Murasugi sum provided the addition is done along minimal genus surfaces. Taking the Murasugi sum of two knots can thus be considered a "natural" operation on pairs consisting of knots together with minimal genus Seifert surfaces. However, the 

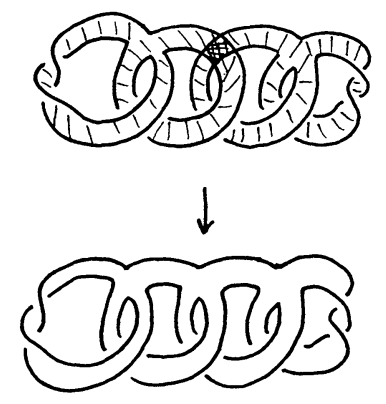

$\downarrow$

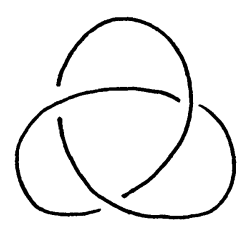

FIGURE 1
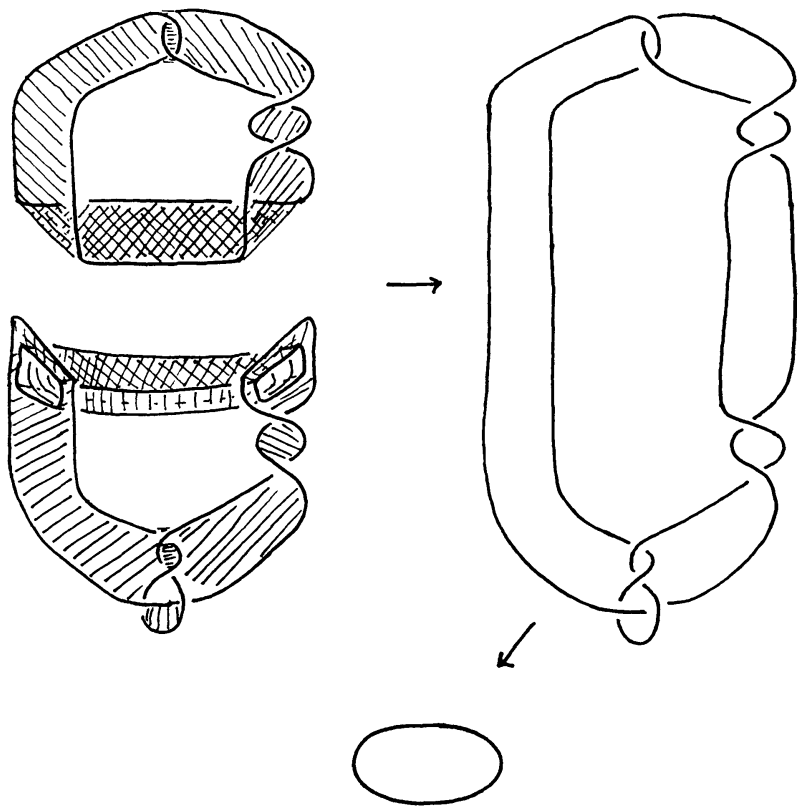

FIGURE 2

operation of constructing a Murasugi sum is not confined to minimal genus or even incompressible Seifert surfaces; we give two examples to illustrate that the genus does not behave in a predictable way in this larger category. The first [Figure 1] is an example of two trivial knots, 
each bounding a (compressible) genus one surface, summed along a square to yield a trefoil. The second example [Figure 2] is two figure eight knots, one bounding a genus one surface and the other bounding a (compressible) genus two surface, summed along a square to yield the trivial knot.

\section{REFERENCES}

[G1] D. Gabai, Genus is superadditive under band connected sum, Topology, 26, No. 2 (1987), 209-210.

[G2] _- The Murasugi sum is a natural geometric operation, Contemp. Math., vol. 20, Amer. Math. Soc., Providence, RI, 1983, pp. 131-143.

[S] M. Scharlemann, Sutured manifolds and generalized Thurston norms, J. Differential Geom., 29 (1989), 557-614.

[Sc] H. Schubert, Uber eine numerische Knoteninvariante, Math. Z., 61 (1954), 245-288.

Received April 4, 1992. Partially supported by an NSF grant and a grant from the Alfred P. Sloan Foundation.

UNIVERSITY OF CALIFORNIA

DAvis, CA 95616 



\section{CONTENTS}

D. Bisch, A note on intermediate subfactors , . , . . . . . . . . . . . . . . . . 201

M. J. Carro and J. Soria, Tent spaces over general approach regions and pointwise

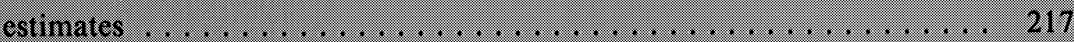

J. A. Charris, M. E. H. Ismail, and S. Monsalve, On sieved orthogonal polynomials X: general blocks of recurrence relations . . . . . . . . . . . . . . . . 237

K. S. Chou and T. Y. H. Wan, Asymptotic radial symmetry for solutions of $\Delta u+$ $e^{\prime \prime}=0$ in a punctured disc . . . . . . . . . . . . . . . . . . . . . . . 269

M. E. Fogel, Knots with algebraic unknotting number one . . . . . . . . . . . . . 277

K. N. Jones, The structure of closed nonpositively curved Euclidean cone 3-manifolds . . . . . . . . . . . . . . . . . . . . . . . . . . . . . . . . 297

M. Kaneda, On the Frobenius morphism of flag schemes . . . . . . . . . . 315

H. T. Kaptanoğlu, Möbius-invariant Hilbert spaces in polydiscs . . . . . . . . . 337

K. Kuribayashi, The cohomology ring of the spaces of loops on Lie groups and homogeneous spaces ............................ 361

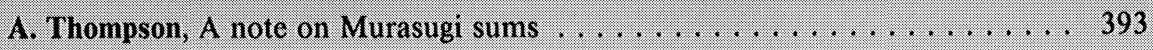




\title{
PACIFIC JOURNAL OF MATHEMATICS
}

\author{
Volume $163 \quad$ No. $2 \quad$ April 1994
}

A note on intermediate subfactors

201

DIETMAR BISCH

Tent spaces over general approach regions and pointwise estimates

217

MARÍA J. CARRO and JAVIER SORIA

On sieved orthogonal polynomials. X. General blocks of recurrence

237 relations

JAIro A. Charris, Mourad ISMAIL and SERGio Monsalve

Asymptotic radial symmetry for solutions of $\Delta u+e^{u}=0$ in a punctured disc

Kai Seng (Kaising) Chou (Tso) and Tom YaU-Heng Wan

Knots with algebraic unknotting number one

MicAh Elton Fogel

The structure of closed non-positively curved Euclidean cone

3-manifolds

KERRY NELSON JONES

On the Frobenius morphism of flag schemes

MASAHARU KANEDA

Möbius-invariant Hilbert spaces in polydiscs

H. TuRgay KaptanOGLU

The cohomology ring of the spaces of loops on Lie groups and homogeneous spaces

KATSUHIKO KURIBAYASHI

A note on Murasugi sums

ABIGAIL A. THOMPSON 